\title{
Risco Cardiovascular em Usuários de Programa de Atenção a Hipertensos e Diabéticos em um Município do Paraná-Brasil
}

\section{Riesgo Cardiovascular en Usuarios del Programa de Atención de Hipertensos y Diabéticos en una Ciudad de la Provincia del Paraná-Brasil \\ Cardiovascular Risk in Users of Hypertensive and Diabetic Attention Program in a City of Paraná-Brazil}

Recibido: 17 de Nov. 2013/Enviado para modificación: 16 de Ene. 2013/Aceptado: 27 de Feb. 2014

\author{
Tatiane Baratieri ${ }^{1}$ \\ Universidade Estadual do Centro-Oeste - Unicentro \\ Jéssica Dal Santo Ottoni ${ }^{2}$ \\ Universidade Estadual do Centro-Oeste - Unicentro \\ Maria Luciana Botti \\ Universidade Estadual do Centro-Oeste - Unicentro \\ Rita de Cássia Serpa Maicel ${ }^{4}$ \\ Universidade Estadual do Centro-Oeste - Unicentro \\ Letícia Gramazio Soares \\ Universidade Estadual do Centro-Oeste - Unicentro
}

\section{RESUMEN}

Introdução: As Doenças Crônicas Não Transmissíveis constituem-se em um dos principais problemas de saúde pública no mundo, quando associadas a fatores de risco aumentam as chances de ocorrência de um evento cardiovascular. Objetivo: Caracterizar o perfil sociodemográfico, os fatores de risco cardiovascular associados e a estratificação de risco a partir da análise das fichas cadastrais de usuários adultos do Programa Hiperdia. Materiais e métodos: Trata-se de estudo descritivo-exploratório, documental e quantitativo, em município de pequeno porte na região centro-sul do Estado do Paraná. Os dados foram coletados a partir de 62 fichas de usuários com até 60 anos cadastrados no Programa Hiperdia. Resultados: Quanto à caracterização dos participantes do estudo, o $84 \%$ é do sexo feminino, $52 \%$ casados; $85 \%$ estão na faixa etária de 40 a 59 anos; $66 \%$ considera pertence a raça brancos e $68 \%$ cursou ensino fundamental. Sobre a estratificação de risco verificou-se que $51 \%$ apresenta risco alto ou muito alto para evento cardiovascular. Sobre fatores de risco cardiovascular $39 \%$ tem antecedentes familiares (cardiovascular), $27 \%$ é diabético, 29\% tabagista, $47 \%$ é sedentário, $66 \%$ está com sobrepeso/obesidade e $100 \%$ é hipertenso. Conclusão: O grau de risco aumenta conforme aumenta a idade, sendo que sobrepeso/obesidade, diabetes mellitus e sedentarismo são os principais fatores de risco determinantes do grau de risco. É importante a identificação dos fatores de risco presentes nas pessoas com hipertensão e diabetes de forma a direcionar o cuidado, bem como, a partir desses, realizar a estratificação de risco dos usuários, a fim de atender cada pessoa conforme suas necessidades de saúde, viabilizando a prevenção de agravos.

Palavras Chave: Hipertensão, Diabetes Mellitus, fatores de risco, doença crônica (Fuente: DeCS).

\section{ABSTRACT}

Introduction: Diseases chronic not communicable constitute one of the major public health problems in the world when the associated risk factors increase the chances of occurrence of a cardiovascular event. Objective: To characterize the cardiovascular risk factors and risk stratification by analyzing registration forms of adult users Hiperdia Program. Materials and methods: This is a descriptive exploratory study, document and quantitative, in a small city in the south-central region of the state of Paraná. Data were collected from 62 users chips with up to 60 years enrolled in the program Hiperdia. Results: The study population was characterized by $84 \%$ female, $52 \%$ married, $85 \%$ aged $40-59$ years, $66 \%$ white and $65 \%$ with elementary education. About risk stratification was found $51 \%$ have high or very high risk for cardiovascular events. About risk factors of cardiovascular $39 \%$ have a family history (cardiovascular), $27 \%$ are diabetic, $29 \%$ smokers, $47 \%$ are sedentary, $66 \%$ are overweight/obesity and $100 \%$ is hypertensive. Conclusion: The degree of risk increases with increasing age, and overweight/obesity, diabetes and physical inactivity are the main risk factors that determine the degree of risk. It is important to identify the risk factors that people with hypertension and diabetes are exposed to direct care, as well as from those factors, perform risk stratification of users in order to meet each person according to their health needs, enabling the prevention of injuries.

Keywords: Hypertension, Diabetes Mellitus, risk factors, chronic disease (Source: MeSH, NLM)

Para citar este artículo: Baratieri T, Ottoni J, Botti ML, Serpa MR, Gramazio SL. Risco cardiovascular em usuários de programa de atencao a hipertensos e diabéticos em um município do Paraná-Brasil. Cienc. innov. salud. 2014; 2 (1): 18-26.

1 Enfermeira. Mestre em Enfermagem. Docente Assistente A do Departamento de Enfermagem da Universidade Estadual do Centro-Oeste - Unicentro. Paraná, Brasil. Email: baratieri.tatiane@gmail.com)

Enfermeira. Pós-graduanda da especialização em Gestão dos Serviços de Saúde pela Universidade Aberta do Brasil, ofertada pela Unicentro.

Enfermeira. Mestre em Enfermagem. Docente Assistente A do Departamento de Enfermagem da Unicentro.

Enfermeira. Professor Auxiliar da Unicentro e Enfermeira da Secretaria Municipal de Saúde de Guarapuava/PR.

Enfermeira. Mestre em Enfermagem. Docente do Departamento de Enfermagem da Unicentro. 


\section{Introdução}

Até a metade do século $\mathrm{XX}$, as doenças que causavam a morte dos indivíduos e mobilizava os profissionais de saúde eram as infectocontagiosas. As transformações decorrentes da transição demográfica, epidemiológica, nutricional e ocupacional geraram alterações importantes no que diz respeito ao processo saúde-doença, pois provocaram mudanças nos hábitos de vida das pessoas, tornado-as menos saudáveis $(1,2)$.

Atualmente, as Doenças Crônicas NãoTransmissíveis (DCNT) constituem em um fenômeno global, de modo que a magnitude desse aumento em incidência e prevalência aponta que as maiores proporções mundiais de óbitos por esses agravos ocorrem em países em desenvolvimento (3).

No Brasil, a principal causa de morbidade, bem como, de mortalidade são as DCNT. Em 2007, foram responsáveis por $72 \%$ das mortes no país, destacandose a hipertensão arterial sistêmica (HAS) e diabetes mellitus (DM), e a relação dessas com os índices de mortalidade por doenças cardiovasculares como infarto agudo do miocárdio, acidente vascular cerebral, insuficiência cardíaca e coronariana, insuficiência renal crônica entre outras (3), impactando negativamente na vida das pessoas.

O presente estudo aborda os fatores de risco cardiovascular em pessoas com hipertensão e/ou diabetes, no contexto de um programa de atenção à saúde do Brasil. Assim, dentre os fatores de risco cardiovascular pode-se citar o tabaco, a obesidade, o sedentarismo, o baixo consumo de frutas, verduras e legumes, o alcoolismo, que quando associados à hipertensão arterial e diabetes potencializam a ocorrência de um evento cardiovascular $(1,2)$.

Estudos revelam que a hipertensão arterial quando acompanhada de fatores de risco está relacionada à maior incidência de outras doenças como, aterosclerose, cardiopatia isquêmica, acidente cerebrovascular e doenças vasculares, tanto renais quanto periférica (4).

A concepção de que os fatores de risco para doenças crônicas afetam somente indivíduos em idade avançada, ainda está muito presente entre as pessoas, 0 que contribui para a existência de fatores entre adultos jovens em idade laboral. A literatura aponta $65 \%$ dos óbitos por doenças crônicas nesta população, ainda aponta que são responsáveis por $40 \%$ das aposentadorias precoces (5).

Existem evidências científicas de que atuar sobre os fatores de risco pode eliminar pelo menos $80 \%$ das doenças cardiovasculares e diabete tipo 2. Um conjunto pequeno de fatores de risco é responsável pela maioria das mortes por doenças crônicas e por fração substancial da carga de doenças devido a essas enfermidades (6).

Tendo em vista então as mudanças no processo de adoecimento da população, no século $\mathrm{XX}$ foi implementado no Brasil por meio da Portaria $\mathrm{n}^{\circ}$ 371/GM (7), em 4 de março de 2002, o Programa HiperDia, que se configura em um Plano de Reorganização da Atenção à Hipertensão Arterial e Diabetes Mellitus, que estabelece metas e diretrizes para ampliar ações de prevenção, diagnóstico, tratamento e controle dessas doenças, mediante a reorganização do trabalho de atenção à saúde.

Portanto, o Hiperdia surgiu como uma reorganização da atenção a estas doenças, uma maneira de acompanhar as transformações ocorridas nesta população, além de descrever suas características (8).

Este Programa prevê um Sistema de Cadastramento e Acompanhamento de Hipertensos e Diabéticos, o SisHiperdia, que é um instrumento que serve para nortear as investigações das DCNT e disponibilizar informações relacionadas ao impacto epidemiológico no contexto brasileiro (9).

Tendo em vista a incidência de DCNT entre adultos jovens, a existência de fatores de risco preveníveis nesta população, bem como os desdobramentos destas doenças para a sociedade e serviços de saúde, optou-se por realizar esta pesquisa. $\mathrm{O}$ estudo teve como objetivo geral analisar as fichas cadastrais de usuários adultos do Programa Hiperdia e como objetivos específicos: (i) levantar o perfil sociodemográfico destes usuários; (ii) caracterizar os fatores de risco para eventos cardiovasculares e (iii) realizar a estratificação de risco cardiovascular dos usuários.

\section{Materiais e Métodos}

Trata-se de uma pesquisa de caráter descritivoexploratória, documental de abordagem quantitativa. A pesquisa foi realizada no Brasil, no município de Pitanga, localizado no centro-sul do Estado do Paraná. 
O município possui oito equipes de Estratégia Saúde da Família (ESF), com 100\% de cobertura, sendo que dessas, apenas duas unidades fizeram parte do presente estudo, tendo em vista que eram as equipes de saúde que estavam com os cadastros dos usuários no Hiperdia atualizados.

A seleção da amostra que compôs o estudo seguiu os critérios de inclusão: fichas de usuários cadastrados no Hiperdia nas equipes de saúde selecionadas; usuários adultos, com menos de 60 anos, cadastrados no Hiperdia das equipes de saúde selecionadas, e excluídas as fichas de cadastro com informações sem preenchimento. Assim, nas equipes de saúde pesquisadas havia 206 usuários cadastrados no Hiperdia, porém, desses, apenas 62 cadastros atenderam aos critérios de inclusão.

A coleta de dados se deu em abril de 2012. Os dados para caracterização sociodemográfica e de saúde foram: sexo; estado civil; faixa etária (classificada conforme a ficha de cadastro no hiperdia); raça/cor; escolaridadade; valor da pressão arterial no momento do cadastro do usuário. Além disso, a partir da ficha cadastral foram identificados os fatores de risco cardiovascular e doenças associadas: antecedentes familiares de evento cardiovascular, hipertensão arterial, diabetes mellitus tipo I e II, tabagismo, sedentarismo, sobrepeso/obesidade (ou seja, Índice de Massa Corporal igual ou maior de $25 \mathrm{Kg} / \mathrm{m}^{2}$ ) (10), outras complicações (infarto agudo no miocárdio, outras coronariopatias, acidente vascular encefálico, pé diabético, amputação por diabetes, doença renal).

Com base nos valores pressóricos, fatores de risco cardiovascular e outras complicações identificadas, a população em estudo foi classificada, pelos pesquisadores, conforme o grau de risco seguindo a tabela de Risco Estratificado e Quantificação de Prognóstico - Pressão Arterial (mmHg) (11), que consta na ficha de cadastro do Hiperdia, apresentada a seguir em forma de quadro em quadro 1 :

Quadro 1. Classificação de risco estratificado e quantificação de prognóstico (11).

\begin{tabular}{|c|c|c|c|}
\hline $\begin{array}{c}\text { Outros fatores } \\
\text { de risco ou } \\
\text { doença }\end{array}$ & $\begin{array}{c}\text { Grau 1 } \\
\text { Hipertensão } \\
\text { leve PAS 140- } \\
159 \text { ou PAD } \\
90-99\end{array}$ & $\begin{array}{c}\text { Grau 2 } \\
\text { Hipertensão } \\
\text { moderada } \\
\text { PAS 160-179 } \\
\text { ou PAD 100- } \\
109\end{array}$ & $\begin{array}{c}\text { Grau 3 } \\
\text { Hipertensão } \\
\text { grave } \\
\text { PAS }>/=180 \text { ou } \\
\text { PAD }>/=110\end{array}$ \\
\hline $\begin{array}{l}\text { I-Sem outros } \\
\text { fatores de risco }\end{array}$ & Risco baixo & Risco médio & Risco alto \\
\hline
\end{tabular}

\begin{tabular}{|c|c|c|c|}
\hline $\begin{array}{l}\text { Outros fatores } \\
\text { de risco ou } \\
\text { doença }\end{array}$ & $\begin{array}{c}\text { Grau 1 } \\
\text { Hipertensão } \\
\text { leve PAS 140- } \\
159 \text { ou PAD } \\
90-99\end{array}$ & $\begin{array}{c}\text { Grau 2 } \\
\text { Hipertensão } \\
\text { moderada } \\
\text { PAS 160-179 } \\
\text { ou PAD 100- } \\
109\end{array}$ & $\begin{array}{c}\text { Grau 3 } \\
\text { Hipertensão } \\
\text { grave } \\
\text { PAS }>/=180 \text { ou } \\
\text { PAD }>/=110\end{array}$ \\
\hline $\begin{array}{l}\text { II- 1-2 Fatores } \\
\text { de risco }\end{array}$ & Risco médio & Risco médio & $\begin{array}{l}\text { Risco muito } \\
\text { alto }\end{array}$ \\
\hline $\begin{array}{l}\text { III- } 3 \text { ou mais } \\
\text { fatores de risco } \\
\text { ou lesões nos } \\
\text { órgãos-alvo ou } \\
\text { diabetes. }\end{array}$ & Risco alto & Risco alto & $\begin{array}{l}\text { Risco muito } \\
\quad \text { alto }\end{array}$ \\
\hline $\begin{array}{l}\text { IV- Condições } \\
\text { clínicas } \\
\text { associadas, } \\
\text { incluindo doença } \\
\text { cardiovascular } \\
\text { ou renal. }\end{array}$ & $\begin{array}{l}\text { Risco muito } \\
\quad \text { alto }\end{array}$ & $\begin{array}{l}\text { Risco muito } \\
\quad \text { alto }\end{array}$ & $\begin{array}{l}\text { Risco muito } \\
\quad \text { alto }\end{array}$ \\
\hline
\end{tabular}

Para realizar a estratificação de risco, cada caso é analisado isoladamente, no qual se analisa os fatores de risco e critérios que o usuário apresenta, com o objetivo de classificá-lo em um grupo de risco dentre quatro disponíveis, obedecendo ao grau de complexidade do caso, conforme ao quadro apresentada acima.

Os grupos de risco disponíveis para classificação bem como os critérios analisados na estratificação de risco são: (1) Grupo de baixo risco: homens e mulheres menores de 55 e 65 anos respectivamente, com HAS de grau I e sem fatores de risco; (2) Grupo de risco médio: indivíduos portadores de hipertensão grau 1 ou 2, com um ou dois fatores de risco cardiovascular. Pode ainda, apresentar baixos níveis de pressão arterial, mas possuir múltiplos fatores de risco. E ainda, possuir altos níveis pressóricos e nenhum fator de risco, ou um a dois fatores de risco; (3) Grupo de risco alto: portadores de HAS grau I ou II associados a três ou mais fatores de risco associados, ou eram portadores de hipertensão grau 3 sem outros fatores de risco; (4) Grupo de risco muito alto: portadores de HAS grau 3 e que possuem um ou mais fatores de risco associados, com doença renal ou cardiovascular já manifesta (11).

Após a coleta, as informações obtidas foram transcritas e tabuladas com auxílio do Editor Estatístico Excel versão Microsoft Windows 2007. Além disso, foi utilizado o software estatístico SPSS versão 15.0, e o nível de significância foi estabelecido em $5 \%$. As variáveis foram apresentadas e descritas em frequência relativa e absoluta. 
Foi empregado o teste de correlação de Pearson, que mede o grau de correlação entre variáveis, de modo que o coeficienteo $\mathrm{r}$ superior a 0,70 indica forte correlação. Além disso, as variáveis de antecedentes familiares, diabetes mellitus, tabagismo, IMC e presença de complicações foram testadas individualmente, através do teste Qui-quadrado (X2), quanto à sua influência na determinação do risco. Devido ao baixo número de observações, utilizou-se a correção de Yates para adequação do modelo. A discussão dos dados deu-se com base na literatura disponível sobre o tema.

A pesquisa foi encaminhada para análise e aprovação do Comitê de Ética e Pesquisa da Universidade Estadual do Centro Oeste, conforme prerrogativas da Resolução n. ${ }^{\circ}$ 466/2012 do Conselho Nacional de Saúde do Brasil, que regulamenta as pesquisas que envolvem seres humanos, com aprovação pelo parecer de número 082/2012.

\section{Resultados}

Quanto ao perfil sociodemográfico (Tabela 1) da população do estudo, verifica-se $84 \%$ (52) é do sexo feminino e $52 \%$ (32) casados. Sobre a faixa etária, $85 \%$ (53) dos sujeitos estão entre 40 a 59 anos. Quanto à etnia, 66\% (41) dos cadastrados são brancos. Na análise da variável escolaridade, evidenciou-se uma predominância de 42 (68\%) de indivíduos com ensino fundamental.

Tabela 1. Perfil sociodemográfico dos usuários cadastrados no Hiperdia. ( $n=62)$. Pitanga, PR, Brasil, 2012.

\begin{tabular}{llcc}
\hline Variável & & Frec. Obs. & Rep .\% \\
\hline \multirow{2}{*}{ Sexo } & Feminino & $\mathbf{5 2}$ & $\mathbf{8 4}$ \\
& Masculino & $\mathbf{1 0}$ & $\mathbf{1 6}$ \\
\hline \multirow{4}{*}{ Estado } & Solteiro & $\mathbf{8}$ & $\mathbf{1 3}$ \\
civil & Casado & $\mathbf{3 2}$ & $\mathbf{5 2}$ \\
& Viúvo & $\mathbf{9}$ & $\mathbf{1 4}$ \\
& Divorciado & $\mathbf{8}$ & $\mathbf{1 3}$ \\
& Mora com companheiro & $\mathbf{5}$ & $\mathbf{8}$ \\
\hline \multirow{2}{*}{ Faixa } & 20 a 29 & $\mathbf{1}$ & $\mathbf{2}$ \\
etária & 30 a 39 & $\mathbf{8}$ & $\mathbf{1 3}$ \\
(anos) & 40 a 49 & $\mathbf{2 0}$ & $\mathbf{3 2}$ \\
& 50 a 59 & $\mathbf{3 3}$ & $\mathbf{5 3}$ \\
\hline \multirow{3}{*}{ Raça/Cor } & Branca & $\mathbf{4 1}$ & $\mathbf{6 6}$ \\
& Parda & $\mathbf{1 4}$ & $\mathbf{2 3}$ \\
& Preta & $\mathbf{7}$ & $\mathbf{1 1}$ \\
\hline \multirow{2}{*}{ Escolarid } & Não alfabetizado & $\mathbf{5}$ & $\mathbf{8}$ \\
ade & Ensino Fundamental & $\mathbf{4 2}$ & $\mathbf{6 8}$ \\
& Ensino Médio & $\mathbf{9}$ & $\mathbf{1 5}$ \\
& Ensino Superior I & $\mathbf{6}$ & $\mathbf{9}$ \\
\hline
\end{tabular}

Cienc. innov. salud. Junio 2014; 2 (1):18 - 26. Universidad Simón Bolívar (Col). ISSN: 2344-8636 http://portal.unisimonbolivar.edu.co:82/rdigital/innovacionsalud
A Tabela 2 apresenta forte correlação entre idade e grau de risco, sendo que todas as variáveis apresentaram significância estatística $(p \leq 0,05)$. Evidencia-se que quanto maior a idade maior é o surgimento de risco, além disso, os dados reportam que conforme o aumento da idade o grau de risco também aumenta, sendo que a maioria das pessoas entre 50-59 anos encontram-se com grau de risco alto, $14(23 \%)$, ou muito alto, $5(8 \%)$, representando a maioria, $19(58 \%)$ das pessoas na referida faixa etária.

Evidencia-se que 39\% (24) dos indivíduos pesquisados estão com risco alto para agravo cardiovascular e sete (12\%) com risco muito alto, correspondendo à maioria dos adultos pesquisados.

Tabela 2. Distribuição dos hipertensos e/ou diabéticos, conforme relação entre classificação de risco e faixa etária. (n=62). Pitanga, PR, Brasil, 2012.

\begin{tabular}{|c|c|c|c|c|c|c|}
\hline \multirow{2}{*}{\multicolumn{2}{|c|}{$\begin{array}{l}\text { Faixa } \\
\text { etária }\end{array}$}} & \multicolumn{4}{|c|}{ Grau de risco } & \multirow[b]{2}{*}{ Total } \\
\hline & & Baixo & Médio & Alto & $\begin{array}{c}\text { Muito } \\
\text { Alto }\end{array}$ & \\
\hline \multirow{2}{*}{$20-29$} & $\mathrm{n}$ & 1 & - & - & - & 1 \\
\hline & $\%$ & 2 & - & - & - & 2 \\
\hline \multirow{2}{*}{$30-39$} & $\mathrm{n}$ & 2 & 1 & 3 & 2 & 8 \\
\hline & $\%$ & 3 & 2 & 5 & 3 & 13 \\
\hline \multirow{2}{*}{$40-49$} & $\mathrm{n}$ & 8 & 5 & 7 & 0 & 20 \\
\hline & $\%$ & 13 & 8 & 11 & 0 & 32 \\
\hline \multirow{2}{*}{$50-59$} & $\mathrm{n}$ & 10 & 4 & 14 & 5 & 33 \\
\hline & $\%$ & 16 & 6 & 23 & 8 & 53 \\
\hline \multirow{2}{*}{ Total } & $\mathrm{n}$ & 21 & 10 & 24 & 7 & - \\
\hline & $\%$ & 33 & 16 & 39 & 12 & - \\
\hline \multicolumn{2}{|c|}{$r^{*}$} & 0,96 & 0,87 & 0,98 & 0,71 & - \\
\hline \multicolumn{2}{|c|}{ p-Valor } & 0,00072 & 0,00175 & 0,00047 & 0,00494 & - \\
\hline
\end{tabular}

*r: coeficiente de correlação de Pearson superior a 0,70 representa forte correlação.

Ao tratar sobre os fatores de risco cardiovasculares prevalentes na população pesquisada (Tabela 3), a presença de antecedentes familiares está em 39\% (24) da população cadastrada.

Com relação ao fator de risco diabetes, $27 \%$ (17) dos indivíduos apresenta essa doença. Ao realizar o levantamento sobre a amostra total de fumantes, pode- 
se observar que $29 \%$ (18) dos participantes apresenta o referido fator de risco.

Com relação ao sedentarismo está presente em $47 \%$ (29) da população pesquisada. Outro dado relevante foi com relação ao sobrepeso/obesidade dos sujeitos do estudo, sendo que 66\% (41) estão com peso acima do ideal.

Ao analisar a relação entre fatores de risco e a estratificação de risco dos pesquisados, comparado o valor crítico de Qui-quadrado $\left(\chi^{2} \mathrm{c}\right)$ igual a 7.815, com grau três de liberdade, aos valores de Qui-quadrado calculados para os indicadores de risco (Tabela 3), observa-se que todos se confirmam na definição do grau de risco; entretanto, apenas os fatores de risco como antecedentes familiares e presença de complicações apresentaram significância estatística $(\mathrm{p} \leq 0,05)$.

Tabela 3. Distribuição dos hipertensos e/ou diabéticos, conforme a relação entre fatores de risco cardiovascular e grau de risco (n=62). Pitanga, PR, Brasil, 2012.

\begin{tabular}{|c|c|c|c|c|c|c|c|c|c|c|c|c|c|}
\hline \multirow{2}{*}{\multicolumn{2}{|c|}{ Fatores de risco }} & \multicolumn{2}{|c|}{ Risco baixo } & \multicolumn{2}{|c|}{ Risco médio } & \multicolumn{2}{|c|}{ Risco alto } & \multicolumn{2}{|c|}{ Risco muito alto } & \multicolumn{2}{|c|}{ Total } & \multirow{2}{*}{$\chi^{2}$} & \multirow{2}{*}{$\begin{array}{c}\text { Valor de } \\
\text { p }\end{array}$} \\
\hline & & Frec. Obs. & Rep. \% & Frec. Obs. & Rep. \% & Frec. Obs. & Rep. \% & Frec. Obs. & Rep. \% & Frec. Obs. & Rep. \% & & \\
\hline \multirow{2}{*}{$\begin{array}{l}\text { Antecedentes } \\
\text { familiares }\end{array}$} & Sim & -- & -- & 2 & 20 & 17 & 71 & 5 & 71 & 24 & 39 & \multirow{2}{*}{24,6} & \multirow{2}{*}{0,05} \\
\hline & Não & 21 & 100 & 8 & 80 & 7 & 29 & 2 & 29 & 38 & 61 & & \\
\hline \multirow{2}{*}{$\begin{array}{l}\text { Diabetes } \\
\text { Mellitus }\end{array}$} & Sim & -- & -- & -- & -- & 20 & 83 & 7 & 100 & 17 & 27 & \multirow{2}{*}{43,32} & \multirow{2}{*}{0,9} \\
\hline & Não & 21 & 100 & 10 & 100 & 4 & 17 & - & - & 45 & 73 & & \\
\hline \multirow{2}{*}{ Tabagismo } & Sim & -- & -- & 4 & 40 & 11 & 46 & 3 & 43 & 18 & 29 & \multirow{2}{*}{10,48} & \multirow{2}{*}{0,6} \\
\hline & Não & 21 & 100 & 6 & 60 & 13 & 54 & 4 & 57 & 44 & 71 & & \\
\hline \multirow{2}{*}{ Sedentarismo } & Sim & -- & -- & 4 & 40 & 22 & 92 & 3 & 43 & 29 & 47 & \multirow{2}{*}{34,33} & \multirow{2}{*}{0,6} \\
\hline & Não & 21 & 100 & 6 & 60 & 2 & 8 & 4 & 57 & 33 & 53 & & \\
\hline \multirow{2}{*}{ IMC } & $\begin{array}{l}\text { Sobrepeso/ } \\
\text { Obesidade }\end{array}$ & -- & -- & 10 & 100 & 24 & 100 & 7 & 100 & 41 & 66 & \multirow{2}{*}{56,88} & \multirow{2}{*}{0,1} \\
\hline & Normal & 21 & 100 & -- & -- & -- & -- & -- & -- & 21 & 34 & & \\
\hline \multirow{2}{*}{$\begin{array}{c}\text { Presença de } \\
\text { complicações }\end{array}$} & Sim & -- & -- & -- & -- & 7 & 29 & 7 & 100 & 14 & 23 & \multirow{2}{*}{28,72} & \multirow{2}{*}{0,001} \\
\hline & Não & 21 & 100 & 10 & 100 & 17 & 71 & -- & -- & 48 & 77 & & \\
\hline
\end{tabular}

\section{Discusión}

Ao tratar sobre o perfil sociodemográfico dos participantes do estudo, os achados, em relação ao sexo, corroboram com a literatura, que aponta maior prevalência de fatores de risco para DCNT na população feminina $(12,13)$. Dados semelhantes são apontados em outros estudos, também em relação à situação conjugal, havendo prevalência do estado civil casado (14).

Salienta-se que a família é considerada um fator positivo para o enfrentamento de doenças, pois estimula o tratamento terapêutico, o que aumenta as chances de sucesso e adesão às orientações fornecidas (15).

A presenta de DCNT no presenta estudo é maior de 50 a 59 anos, o que está em conformidade com a literatura sobre o tema $(12,16)$.

No que tange à etnia, houve maior número de participantes brancos, destacando-se que o Paraná foi colonizado por europeus, o que pode esclarecer a predominância da raça branca no estudo, sendo um atributo regional (17). Entretanto, outros estudos apontam que indivíduos negros têm níveis pressóricos mais elevados que os de outras raças, porém vem se observando variações de prevalência da hipertensão em outras etnias $(12,18)$.

Outro dado também encontrado na literatura é a predominância de pessoas com ensino fundamental incompleto (12). Quanto menor o nível de escolaridade maior a chance de se desenvolver a HAS, e mais difícil é o seu controle (18). Este achado é preocupante, pois estes indivíduos requerem um nível de cuidado organizado, tanto nos medicamentos quanto na alimentação, e a baixa escolaridade dificulta a compreensão e consequentemente o processo educação em saúde destes usuários para a promoção do autocuidado e autonomia, impactando na qualidade de vida (8).

$\mathrm{O}$ presente estudo evidenciou que a maioria dos usuários pesquisados apresenta risco alto e muito alto

Cienc. innov. salud. Junio 2014; 2 (1):18 - 26. Universidad Simón Bolívar (Col). ISSN: 2344-8636 http://portal.unisimonbolivar.edu.co:82/rdigital/innovacionsalud 
para evento cardiovascular. A literatura aponta que pessoas classificadas com risco baixo têm menos de $10 \%$ de chances de ocorrência de evento cardiovascular nos próximo 10 anos, já pessoas com risco médio têm de 10 a $20 \%$ de chances, já a pessoa classificada com risco alto e muito alto tem de $20 \%$ a $30 \%$ de chances de serem acometidos por um evento cardiovascular nos próximos 10 anos $(19,20)$.

Sobre a estratificação de risco verificou-se forte correlação entre aumento da idade e risco. Outros estudos evidenciaram dados semelhantes, salientando que o risco cardiovascular aumenta conforme aumenta a idade $(12,14)$.

Os resultados evidenciam então, que a prevalência de fatores de risco cardiovasculares está proporcionalmente relacionada com o processo de envelhecimento. Entretanto, deve-se ressaltar que entre adultos jovens, com faixa etária de 20 a 40 anos, observa-se que é progressivo o aumento da ocorrência de doenças crônicas e a presença de fatores de risco (9).

Os fatores de risco cardiovascular são condições/agravos que predispõe um indivíduo ao risco de um evento cardiovascular $(2,18)$, podendo ser classificados como modificáveis e não modificáveis (21).

Sobre o fator de risco "antecedentes familiares (cardiovascular)", o presente estudo apresentou dados semelhantes à literatura (13,22). Apesar do referido fator de risco não ser modificável, o risco de agravos à saúde pode ser minimizado por meio de estratégias de promoção da saúde e prevenção de agravos.

Sobre a presença de diabetes, o estudo também corrobora com a literatura (23). A diabetes mellitus é uma das principais doenças causadoras de complicações cardiovasculares fatais e não fatais, constituindo-se em um fator de risco não modificável, porém passível de controle (23).

Estudo realizado em Porto Alegre/RS (8), apresentou dados semelhantes à presente pesquisa no que se refere ao fator de risco tabagismo.

O controle do tabagismo seria uma das medidas que mais provocaria impacto na redução dos índices de morbimortalidade por doenças cardiovasculares, pois já é confirmada a sua associação ao aumento das placas de ateroma e o seu efeito no perfil lipídico $(24,25)$. Neste sentido, para a população referida neste estudo, seria de grande relevância a oferta de ações educativas, no sentido de incentivar práticas saudáveis, e, aos tabagistas, direcionar para grupos de apoio, com o intuito de expor riscos e, possivelmente, cessar o uso do tabaco.

No que se refere ao sedentarismo o presente estudo apresentou alta prevalência, corroborando com pesquisa sobre o Hiperdia em uma cidade de Santa Catarina que levantou que $59,7 \%$ dos indivíduos cadastrados eram sedentários, sendo esta realizada com todas as unidades de saúde da família do município participante (26). A literatura aponta que pessoas sedentárias têm $30 \%$ a mais de chances de desenvolver hipertensão, ou agravar seus níveis pressóricos, do que os ativos (26).

É válido ressaltar que estimativas mostram que a falta de atividade física é responsável por $10 \%$ a $16 \%$ dos casos de diabetes e $22 \%$ das doenças isquêmicas, além disso, estes têm influência direta no controle da diabetes e hipertensão $(8,15)$. Frente ao exposto, destaca-se a importância do incentivo por parte dos profissionais de saúde à realização de atividade física aos portadores de HAS e ou DM, considerando os benefícios que esta prática oferece para prevenir e/ou reduzir os agravos cardiovasculares.

Outro fator de risco de destaque é o excesso de peso, o qual se constitui atualmente em um problema de saúde pública (27), e fator predisponente para a hipertensão e para o aumento dos valores pressóricos em indivíduos já hipertensos, salientado que de $20 \%$ a $30 \%$ da prevalência de hipertensão pode ser explicada pela presença de excesso de peso (17).

Os fatores de risco sedentarismo, sobrepeso e obesidade, são os que estão mais associados às complicações crônicas decorrentes da DM e da HAS, principalmente em relação às doenças cardiovasculares (8), dados esses encontrados no presente estudo como principais fatores determinantes do grau de risco.

Pequenas perdas de peso, de $5 \%$ a $10 \%$ do peso inicial, promovem melhora no controle da glicemia, reduzem valores pressóricos e também dos níveis de colesterol (28), ou seja, favorecem o controle dos fatores de risco associados, conforme evidenciado no estudo.

Com relação ao diabetes, a perda de peso melhora os níveis de glicemia capilar em $10 \%$ a $20 \%$. Sendo que estes benefícios podem ser mantidos por até três anos, 
mesmo com um aumento significativo de peso (28), ou seja, os fatores de risco estão relacionados para a redução de risco cardiovascular em pessoas com DCNT.

A literatura aponta que a variação da distribuição de gordura em determinadas partes do corpo é um indicador mais importante de complicações metabólicas do que o valor de índice de massa corporal. Desta forma, a obesidade visceral resulta em inúmeras alterações fisiopatológicas, as quais implicam na resistência do organismo a insulina (29).

Tanto o excesso de peso quanto a adiposidade abdominal, tem sido associados aos elevados níveis pressóricos e a síndrome metabólica em indivíduos com DM tipo II, tornando fundamental o conhecimento desses usuários sobre a patologia, para que possa-lhes ser oferecido um melhor tratamento e controle (29), prevenido as complicações, fator esse que também aumenta o risco cardiovascular, variável não incluída no presente estudo.

Os resultados do presente estudo levam a refletir sobre a necessidade de planejamento na assistência em saúde a fim de prevenir agravos cardiovasculares, diminuindo o grau de risco e as chances de ocorrência de um evento cardiovascular. Além disso, a atenção dos profissionais de saúde precisa aumentar às pessoas com DCNT conforme o aumento da idade.

A estratificação de risco permite determinar a melhor assistência ao usuário, evitando as consequências que tais fatores de risco podem trazer ao mesmo, além de aumentar a resolutividade no âmbito da atenção primária e reduzir custos, não somente pela oferta de serviços a usuários que por vezes não necessitam, mas principalmente pela oferta adequada a quem de fato necessita de determinada tecnologia.

Destaca-se que a população em estudo encontra-se em idade adulta, e a ocorrência de um evento cardiovascular nessa fase da vida traz impactos tanto para o indivíduo e sua família, como para os sistema, gerando custos tanto para o setor de saúde como para a economia do país, frente a perda das pessoas em idade produtiva; sugere complementação de um futuro estudo onde seja contemplado indicadores econômicos e projeções do impacto da doença no sistema de saúde.
O presente estudo apresenta como principal limitação o número da população estudada, não permitindo generalizações, porém os dados encontrados corroboram com a literatura sobre o tema.

Desta forma, destaca-se neste estudo a importância da estratificação de risco como ferramenta de pesquisa, de diagnóstico inicial, avaliação e intervencao do risco para realização do levantamento de fatores de risco cardiovascular, o que permite direcionar o cuidado para a prevenção da ocorrência de evento cardiovascular nos próximos 10 anos, o que impacta em maior qualidade de vida das pessoas em idade adulta com DNCT e menores custos para o país, possibilitando que pessoas com essas doenças possam manter-se ativas no mercado de trabalho. Além disso, os dados apontam para a necessidade de maior atenção às pessoas conforme o aumento da idade."

\section{Referencias}

1. Gersh BJ, Sliwa K, Mayosi BM, Yusuf S. The epidemic of cardiovascular disease in the developing world: global implications. Eur Heart J. 2010; 31 (6): 642-648.

2. Moura EC, Silva SA, Malta DC, Neto OLM. Fatores de risco e proteção para doenças crônicas: vigilância por meio e inquérito telefônico. Cad Saúde Pública. 2011; 27 (3): 486-496.

3. Shimidt MI, Duncan BB, Silva GA, Menezes AM, Monteiro CA, Barreto SM, et al. Chronic non-communicable diseases in Brasil: burden and current challenges. Lancet. 2011; 377 (9781): 1949-1961.

4. Magalhães MEC, Brandão AA, Pozzan R. Prevenção da hipertensão arterial: para quem e quando começar? Rev Bras Hipertens. 2010; 17 (2): 93-97.

5. Nogueira D, Faerstein E, Coeli CM, Chor D, Lopes CS, Werneck GL. Reconhecimento, tratamento e controle da hipertensão arterial: Estudo Pró-Saúde, Brasil. Rev. panam. salud pública. 2010; 27 (2): 103-109.

6. Ministério da Saúde Brasil. Sistema de Planejamento do SUS: Uma construção coletiva: Plano Nacional de Saúde (PNS) 2008/2009-2011. Brasília: Ministério da Saúde; 2010.

7. Ministério da Saúde Brasil. Portaria no 371, de 04 de março de 2002. Brasília: Ministério da Saúde, 2002. Disponível em: 
http://bvsms.saude.gov.br/bvs/saudelegis../gm/20 02/prt0371_04_03_2002_rep.html. Acesso em: 23 mar. 2013.

8. Lima LM, SchwartzE, Muniz RM, Zillmer JGV, Ludtke I. Perfil dos usuários do Hiperdia de três unidades básicas de saúde do sul do Brasil. Rev Gaúcha Enferm. 2011; 32 (2):323-329.

9. Moreira TMM, Gomes EB, Santos JC. Fatores de risco cardiovasculares em adultos jovens com hipertensão arterial e/ou diabetes mellitus. Rev Gaúcha Enferm. 2010; 31(4): 662-669.

10. World Health Organization. Obesity: Preventing and managing the global epidemic - Report of a WHO consultation on obesity. Geneva, 1998.

11. Ministério da Saúde Brasil. Hiperdia - Sistema de cadastramento e acompanhamento de hipertensos e diabéticos: manual de operação. Rio de Janeiro: Ministério da Saúde; 2002.

12. Castoldi EB, Oliveira GP, Régis MA, Silva DA. Prevalência de fatores de risco em hipertensos de uma unidade de saúde de diamantino-MT. REMENFE. 2010; 1(1): 57-62.

13. Giroto E, Andrade SM, Cabrera MA, Ridão EG. Prevalência de fatores de risco para doenças cardiovasculares em hipertensos cadastrados em unidade de saúde da família. Acta sci. Health sci. 2009; 31 (1): 77-82.

14. Castro RAA, Moncau JEC, Marcopito LF. Prevalência de hipertensão arterial sistêmica na cidade de formiga, MG. Arq. Bras. Cardiol. 2007; 88(3): 334-339.

15. Ferreira CLRA, Ferreira MG. Características epidemiológicas de pacientes diabéticos da rede pública de saúde - análise a partir do sistema Hiper Dia. Arq Bras Endocrinol Metab. 2009; 53(1): 80-86.

16. Castro NG, Cunha ER, Santos MDS, Dias RS. Hiperdia: conhecimento da cobertura do programa no Maranhão. Cad. Pesq., São Luís. 2010; 17 (2): 33-42.

17. Pelizari VDZV, Mortean ECM, Conti ACM, Mendonça FF. Práticas de controle e prevenção de danos adotados por portadores de hipertensão arterial de Corumbataí do Sul, Paraná, 2010. UNOPAR Cientifica. 2011; 13 (3): 163-168.

18. Zillmer JGV, Schwartz E, Muniz RM, Lima LM. Avaliação da completude das informações do Hiperdia em uma unidade básica do sul do Brasil. Rev Gaúcha Enferm. 2010; 31 (2): 240-246.
19. Brasil. Cadernos de Atenção Básca ${ }^{\circ} 14$ Prevenção clínica de doença cardiovascular, cerebrovascular e renal crônica. Brasília: Ministério da Saúde. Brasília; 2006.

20. Sociedade Brasileira de Cardiologia/Sociedade Brasileira de Hipertensão/Sociedade Brasileira de Nefrologia. VI Diretrizes Brasileiras de Hipertensão. Arq. Bras. Cardiol. 2010; 95(1): 151

21. Casado L, Vianna LM, Thuler LCS. Fatores de risco para doenças crônicas não transmissíveis no Brasil: uma Revisão Sistemática. Rev Bras Cancerol. 2009; 55(4): 379-388.

22. Martins LN, Souza LS, Silva CF, Machado RS, Silva CEF, Vilagra MM, et al. Prevalência dos fatores de risco cardiovascular em adultos admitidos na unidade de dor torácica em vassouras, RJ. Rev Bras Cardiol. 2011; 24(5): 299-307.

23. Gomes TJO, Silva MVR, Santos AA. Controle da pressão arterial em pacientes atendidos pelo programa Hiperdia em uma Unidade de Saúde da Família. Rev Bras Hipertens. 2010; 17(3): 132139.

24. Mitsunaga JK. Perfil e estilo de vida dos idosos cadastrados no SISHIPERDIA da ESF São Geraldo. São Gotardo/MG. [monografia] Universidade Federal de Minas Gerais. Núcleo de Educação em Saúde Coletiva: Uberaba; 2011.

25. Batista ES, Campos TN, Valente FX, Priore SE, Franceschini SCC, Sabarense CM, et al. Impacto do Tabagismo e Álcool sobre a Composição Corporal de Jovens. Revista Brasileira de Cancerologia. 2011; 57(3): 355-363.

26. Dalacosta FM, Dallacosta H, Nunes AD. Perfil de hipertensos cadastrados no programa Hiperdia de uma unidade básica de saúde. Unoesc \& Ciência - ACBS. 2010; 1(1): 45-52.

27. Mantilla MM. Frecuencia de obesidad y sobrepeso en una Institución de Salud de la Ciudad de Barranquilla. Cienc. innov. salud. 2013; 1 (1): 33-39.

28. Programa Nacional de Combate à Obesidade Portugal. Direcção-Geral da Saúde. Divisão de Doenças Genéticas, Crónicas e Geriátricas: Programa Nacional de Combate à Obesidade. Lisboa: 2005.

29. Vasques AC, Pereira PF, Gomide RM, Batista MC, Campos MT, Sant'Ana LF, et al. Influência do excesso de peso corporal e da adiposidade 
central na glicemia e no perfil lipídico de pacientes portadores de diabetes mellitus tipo 2. Arq Bras Endocrinol Metab. 2007; 51(9): 15161521. 